\title{
Variational Level-Set Segmentation and Tracking of Left Ventricle Using Field Prior
}

\author{
Mariam Afshin ${ }^{a, e}$, Ismail Ben Ayed ${ }^{b}$, Ali Islam ${ }^{c}$, Ian Ross ${ }^{d}$, Terry Peters $^{e, a}$ and Shuo Li ${ }^{b, a}$ \\ ${ }^{a}$ University of Western Ontario, London, ON, Canada \\ ${ }^{b}$ GE Healthcare, London, ON, Canada \\ ${ }^{c}$ St. Joseph's Health Care, London, ON, Canada \\ ${ }^{d}$ London Health Science Centre, London, Ontario \\ ${ }^{e}$ Robarts Research Institute, London, ON, Canada
}

\begin{abstract}
This study investigates a novel method of tracking Left Ventricle (LV) curve in Magnetic Resonance (MR) sequences. The method focuses on energy minimization by level-set curve boundary evolution. The level-set framework allows introducing knowledge of the field prior on the solution. The segmentation in each particular time relies not only on the current image but also the segmented image from previous phase. Field prior is defined based on the experimental fact that the mean logarithm of intensity inside endo and epi-cardium is approximately constant during a cardiac cycle. The solution is obtained by evolving two curves following the Euler-Lagrange minimization of a functional containing a field constraint. The functional measures the consistency of the field prior over a cardiac sequence. Our preliminary results show that the obtained segmentations are very well correlated with those manually obtained by experts. Furthermore, we observed that the proposed field prior speeds up curve evolution significantly and reduces the computation load.
\end{abstract}

Keywords: left ventricle, computer-aided detection, segmentation, tracking, variational methods, level-set, field prior, cardiac magnetic resonance imaging.

\section{INTRODUCTION AND DESCRIPTION OF PURPOSE}

Cardiovascular disease is the leading cause of death worldwide. The Left Ventricle (LV) is the main chamber of heart and its function, pumping the oxygenated blood to entire body, is critical for normal cardiac activity. Segmentation and tracking of LV play an essential role in diagnosing cardiovascular diseases.

Several approaches exist for LV segmentation; in clinical routine, manual segmentation of every single slice is used, which is prohibitively time consuming. Alternatively, there are several automatic methods available in

\footnotetext{
Further author information: (Send correspondence to M.A)

M.A : E-mail: mafshin@uwo.ca, Telephone: (519)697-8323

I.B.A: E-mail: Ismail.BenAyed@ge.com, Telephone: (519)646-6000 ext:64626

Medical Imaging 2011: Image Processing, edited by Benoit M. Dawant, David R. Haynor,

Proc. of SPIE Vol. 7962, 79622D · C 2011 SPIE · CCC code: 1605-7422/11/\$18 - doi: 10.1117/12.877557
}

Copyright 2011 Society of Photo-Optical Instrumentation Engineers. One print or electronic copy may be made for personal use only. Systematic reproduction and distribution, duplication of any material in this paper for a fee or for commercial purposes, or modification of the content of the paper are prohibited.

http://doi.org/10.1117/12.877557 
literature based on thresholding, region-growing, edge detection, clustering ${ }^{1-4}$ and variational techniques such as graph-based, ${ }^{5}$ active contour ${ }^{6}$ and level-set ${ }^{7-9}$ algorithms. However, accurate and fast automatic LV segmentation method is still desired. Since variational techniques do not rely on sharp edges and significant intensity changes, they can be used for the segmentation of LV in MR sequence. As discussed by Freedman et al. in, ${ }^{10}$ many methods rely on matching probability distribution of photometric variables that incorporates learned shape and appearance models for the object of interest. Ben Ayed et al. in $^{7}$ and $^{11}$ used a variational method consists of evolving a curve toward region of interest boundaries by measuring the conformity of the overlap between some region distribution learned from manual segmentation of the first frame. Some other approaches ${ }^{12}{ }^{13}$ are based on pixel-wise correspondence between the current image and model distribution of photometric and geometric properties of the target object. Yong et al. in ${ }^{14}$ proposed a motion prior using motion manifold learning to track LV, and used Active Shape Model to obtain the boundaries. Lynch et al. in ${ }^{15}$ presented a coupled level-set segmentation for tracking endo and epi-cardium boundaries. The boundaries of LV are evolved as zero level-set of a high dimension function and curve evolution stops based on gradient and region information.

All the methods rely on shape prior or distribution matching which are both time consuming, computationally expensive and subject-dependent. We propose a new segmentation method based on field prior to automatically segment and track the LV based on minimizing a variational functional by applying level-set method. The proposed method does not require distribution matching and shape prior and, therefore speeds up the level-set evolution.

The proposed variational level-set energy functional is based on three different terms:

- field prior term: which is defined based on the experimental results that the mean logarithm of intensity inside endo and epi-cardium is approximately constant during a cardiac cycle.

- mean term: which keeps the mean intensity within endo and epi-cardium consistent in all frames for each slice.

- edge indicator term: which biases the curve toward high gradient of intensity.

Endo and epi-cardium boundaries in cardiac MR sequence were tracked by evolving two active contours following the Euler-Lagrange optimization of two functional. Each functional contains three terms. Field prior term has some advantages over the existing methods. It removes the need for shape and overlap prior and prevents from the splitting of endo and epi-cardium boundaries. Also it speeded up segmentation and tracking convergence respectively. 


\section{METHOD}

Let $\mathcal{I}$ be a Cardiac MRI sequence containing $I$ frames ${ }^{*}$, each comprising $J$ slices: ${ }^{\dagger} \mathcal{I}_{i j}: \Omega \subset \Re^{2} \rightarrow \Re^{+}$ with $(i, j) \in[1 \ldots I] \times[1 \ldots J]$. The objective of this study is to automatically delineate endo and epi-cardium boundaries of $\mathrm{LV}$ in a cardiac cycle for $i \in\{2 \ldots I\}$.

Let $\mathbf{I}_{1 j}: j \in\{1 \ldots 10\}$ be a reference frame (the first frame) for which the segmentation of all slices are assumed given. We solve the problem by evolution of two closed parameter curves $\Gamma_{\text {in }}^{i j}, \Gamma_{\text {out }}^{i j}:[0,1] \rightarrow \Omega$ in red and green color respectively. $\Gamma_{i n}^{i j}, \Gamma_{o u t}^{i j}$ are evolved until finding desired endo and epi-cardium boundaries for each image of dataset except images in first frame. The curve evolution equations are obtained by minimization of two functional using Euler-Lagrange minimization.

\subsection{The proposed tracking functionals:}

In this section, we propose an original active curve functional $\mathbf{F}$ for the segmentation of the LV. We classify each $\mathcal{I}_{i j}$ to three regions:

- LV cavity is the region inside the $\Gamma_{i n}^{i j}$,

- LV myocardium is the region between $\Gamma_{i n}^{i j}$ and $\Gamma_{\text {out }}^{i j}$,

- background is the region outside of $\Gamma_{\text {out }}^{i j}$.

The proposed variational level-set energy functional consists of three different terms:

The first term is field prior that constrains the mean logarithm intensity inside endo and epi-cardium to be constant during the cardiac cycle. We learn $f_{\text {in }}^{1 j}$ and $f_{\text {out }}^{1 j}$ from the reference frame $\mathbf{I}$ which is manually segmented. function $f_{i n}^{i j}$ and $f_{\text {out }}^{i j}$ in Field prior are defined as follows:

$$
f_{i n}^{i j}=\frac{\int_{\Gamma_{i n}^{i j}}\left(\log \mathcal{I}_{i, j}\right) d x}{a\left(\mathbf{R}_{\Gamma_{i n}^{i j}}\right)} \quad f_{\text {out }}^{i j}=\frac{\int_{\Gamma_{\text {out }}^{i j}}\left(\log \mathcal{I}_{i, j}\right) d x}{a\left(\mathbf{R}_{\Gamma_{\text {out }}^{i j}}\right)}
$$

where $a\left(\mathbf{R}_{\Gamma^{i j}}\right)$ is the area of the region enclosed within $\Gamma^{i j}$.

The second term is an image mean prior which measures the conformity of the mean of image $\mathcal{I}_{i j}$ inside $\Gamma^{i j}$ to the mean learned from the correspondent slice in the reference frame $\mathbf{I}$.

$$
\mu\left(\mathbf{R}_{\Gamma^{i j}}\right)=\frac{\int_{\Gamma^{i j}}\left(\mathcal{I}_{i, j}\right) d x}{a\left(\mathbf{R}_{\Gamma^{i j}}\right)}
$$

The third term is gradient term $g_{i j}=\frac{1}{1+\left\|\nabla \mathcal{I}_{i j}\right\|^{2}}$, an edge indicator function, which regularizes the curve and biases it toward high image transitions. $c$ is a positive constant to guarantee the curve smoothness.

\footnotetext{
* The number of frames $I$ is typically equal to 20 or 25 .

${ }^{\dagger}$ The number of slices $J$ is typically equal to 10 .
} 
We delineate LV cavity endo-cardium boundary by evolving $\Gamma_{i n}^{i j}$ following the minimization of:

$$
\mathbf{F}\left(\Gamma_{i n}^{i j}\right)=\underbrace{\alpha\left(f_{\text {in }}^{i j}-f_{i n}^{1 j}\right)^{2}}_{\text {field prior }}+\underbrace{\beta\left(\mu\left(\mathbf{R}_{\Gamma_{i n}^{i j}}\right)-\mu\left(\mathbf{R}_{\Gamma_{i n}^{1 j}}\right)\right)^{2}}_{\text {mean cavity }}+\underbrace{\lambda \oint_{\Gamma_{i n}^{i j}}\left(g_{i j}+c\right) d s}_{\text {endo-cardium boundary }}
$$

where $\alpha, \beta$ and $\lambda$ are real positive constants to adjust the contribution of each term. The LV myocardium is the region between the endo and epi-cardium curves. Therefore we also need to find the epicardium curve to fully determine the myocardium. The epi-cardium boundary is extracted by evolving $\Gamma_{\text {out }}^{i j}$ following the minimization of:

$$
\mathbf{F}\left(\Gamma_{\text {out }}^{i j}\right)=\underbrace{\alpha\left(f_{\text {out }}^{i j}-f_{\text {out }}^{1 j}\right)^{2}}_{\text {field prior }}+\underbrace{\beta\left(\mu\left(\mathbf{R}_{\Gamma_{\text {out }}^{i j}}\right)-\mu\left(\mathbf{R}_{\Gamma_{\text {out }}^{1 j}}\right)\right)^{2}}_{\text {mean within epi-cardium }}+\underbrace{\lambda \oint_{\Gamma_{\text {out }}^{i j}}\left(g_{i j}+c\right) d s}_{\text {epi-cardium boundary }}
$$

We obtained the curve evolution equations $\Gamma_{i n}^{i j}$ and $\Gamma_{\text {out }}^{i j}$ by minimization of three terms, field as well as mean and edge detection terms, in $\mathbf{F}\left(\Gamma_{i n}^{i j}\right)$ and $\mathbf{F}\left(\Gamma_{\text {out }}^{i j}\right)$ respectively.

\subsection{Curve Evolution:}

The curve evolution equation of $\Gamma_{i n}^{i j}$ and $\Gamma_{o u t}^{i j}$ are obtained using the Euler-Lagrange descent minimization of $\mathbf{F}\left(\Gamma^{i j}\right)$ :

This yiels:

$$
\frac{\partial \Gamma_{i n}^{i j}}{\partial t}=-\frac{\partial \mathbf{F}\left(\Gamma_{i n}^{i j}\right)}{\partial \Gamma_{i n}^{i j}} \quad \frac{\partial \Gamma_{o u t}^{i j}}{\partial t}=-\frac{\partial \mathbf{F}\left(\Gamma_{o u t}^{i j}\right)}{\partial \Gamma_{o u t}^{i j}}
$$

$$
\begin{aligned}
\frac{\partial \Gamma_{i n}^{i j}}{\partial t}= & \left\{\frac{2 \alpha\left(f_{\text {in }}^{i j}-f_{\text {in }}^{1 j}\right)}{a\left(\mathbf{R}_{\Gamma_{\text {in }}^{i j}}^{i j}\right)}\left(f_{\text {in }}^{i j}-\log \left(\mathcal{I}_{i j}\right)\right)+\frac{2 \beta\left(\mu\left(\mathbf{R}_{\Gamma_{i n}^{i j}}\right)-\mu\left(\mathbf{R}_{\Gamma_{\text {in }}^{1 j}}\right)\right)}{a\left(\mathbf{R}_{\Gamma_{\text {in }}^{i j}}^{i j}\right)}\left(\mu\left(\mathbf{R}_{\Gamma_{\text {in }}}^{i j}\right)-\mathcal{I}_{i j}\right)\right. \\
& \left.+\lambda\left[\nabla g_{i j} . n_{\text {in }}^{i j}-\left(g_{i j}+c\right) \kappa_{\text {in }}^{i j}\right]\right\} n_{\text {in }}^{i j} \\
\frac{\partial \Gamma_{\text {out }}^{i j}}{\partial t}= & \left\{\frac{2 \alpha\left(f_{\text {out }}^{i j}-f_{\text {out }}^{1 j}\right)}{a\left(\mathbf{R}_{\Gamma_{\text {out }}^{i j}}^{i j}\right)}\left(f_{\text {out }}^{i j}-\log \left(\mathcal{I}_{i j}\right)\right)+\frac{2 \beta\left(\mu\left(\mathbf{R}_{\Gamma_{\text {out }}^{i j}}\right)-\mu\left(\mathbf{R}_{\Gamma_{\text {out }}^{1 j}}\right)\right)}{a\left(\mathbf{R}_{\Gamma_{\text {out }}^{i j}}^{i j}\right)}\left(\mu\left(\mathbf{R}_{\Gamma_{\text {out }}}^{i j}\right)-\mathcal{I}_{i j}\right)\right. \\
+ & \left.\lambda\left[\nabla g_{\text {ij }} . n_{\text {out }}^{i j}-\left(g_{i j}+c\right) \kappa_{\text {out }}^{i j}\right]\right\} n_{\text {out }}^{i j},
\end{aligned}
$$

where $n^{i j}$ is the outward unit normal vector to $\Gamma^{i j}, \kappa_{i n}^{i j}$ and $\kappa_{\text {out }}^{i j}$ are the mean curvature function of $\Gamma_{i n}^{i j}$ and

$\Gamma_{o u t}^{i j}$, and $\mathbf{R}_{\Gamma_{i n}^{i j}}$ and $\mathbf{R}_{\Gamma_{\text {out }}^{i j}}$ are the region enclosed by $\Gamma_{i n}^{i j}$ and $\Gamma_{o u t}^{i j}$ respectively. The level-set method is used to implement the evolution equation in (6) and (7).

\section{RESULT}

\subsection{Image Acquisition}

A set of 2D short-axis cine magnetic resonance (MR) images of 20 subjects were acquired through the cardiac cycle on a $1.5 \mathrm{~T}$ scanner with fast-imaging employing steady-state acquisition (FIESTA) image sequence mode. The acquisition parameters were as follows: $\mathrm{TR}=2.98 \mathrm{~ms}, \mathrm{TE}=1.2 \mathrm{~ms}$, flip angle $=30$ degree, and slice thickness $=10$ $\mathrm{mm}$. Each subjects dataset consisted of 20 frames, each comprising 10 slices. 


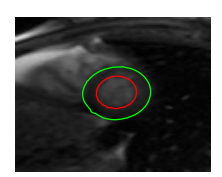

$s 1 f 5$

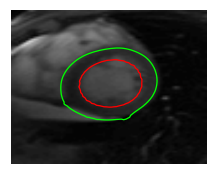

$s 3 f 4$

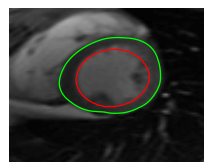

$s 5 f 3$

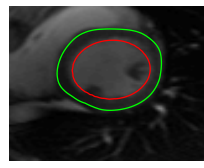

$s 7 f 2$

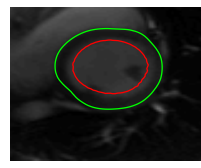

$s 8 f 2$

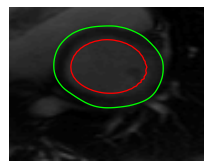

$s 9 f 2$

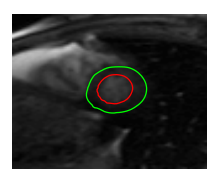

$s 1 f 7$

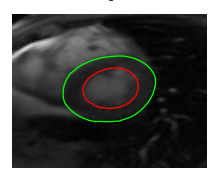

$s 3 f 9$

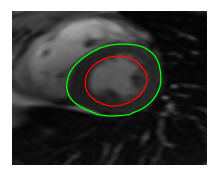

$s 5 f 7$

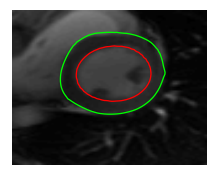

$s 7 f 4$

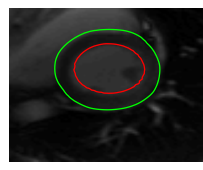

$s 8 f 4$

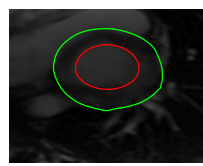

$s 9 f 9$

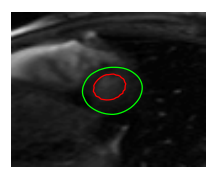

$s 1 f 9$

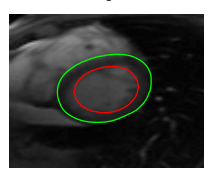

$s 3 f 11$

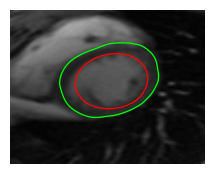

$s 5 f 11$

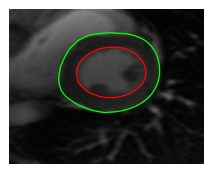

$s 7 f 6$

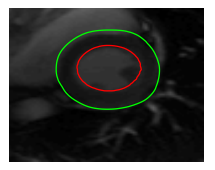

$s 8 f 6$

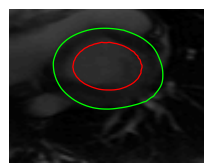

$s 9 f 10$

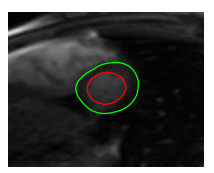

$s 1 f 12$

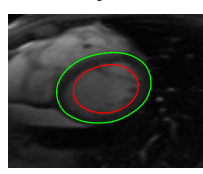

$s 3 f 12$

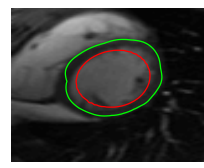

$s 5 f 15$

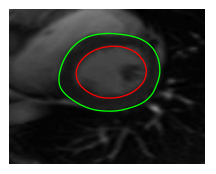

$s 7 f 9$
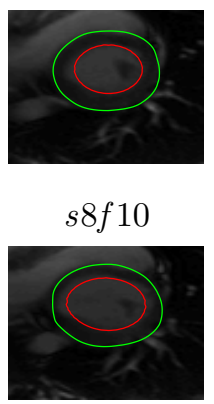

$s 9 f 13$

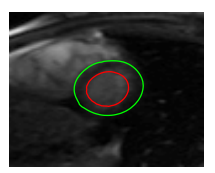

$s 1 f 18$

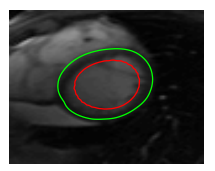

$s 3 f 20$

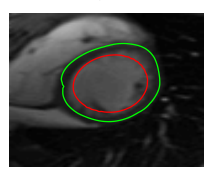

$s 5 f 20$
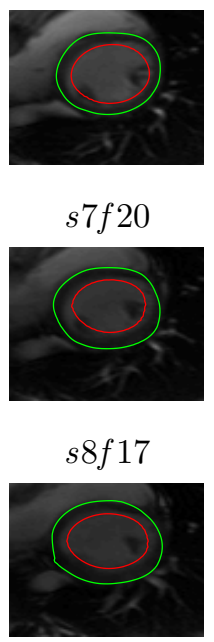

$s 9 f 19$

Figure 1. A representative sample of the segmentation results for one subject in MR sequences. The red curve represents $\Gamma_{i n}$ (the endo-cardium boundary of the LV) and the green curve $\Gamma_{\text {out }}$ (the epi-cardium boundary of LV).

\subsection{Experimental and Statistical Results}

The free parameters were unchanged for all datasets: $\alpha=4, \beta=20, \lambda=0.1, c=5$. Fig. 1 shows a sample of the segmentation results in one subject dataset.

We used the Dice Metric $(D M)$ to measure the similarity between automatic segmentations and manual ones obtained by an expert in all 20 datasets. $D M$ is a statistical value that measures the conformity of two areas. It is a similarity measurement between 0 and 1 . The higher the $D M$, the more accurate the results.

Let $A_{a}, A_{m}$ and $A_{a m}$ be the areas of the automatically detected region, the corresponding manual segmentation obtained by an expert and the overlap between them respectively. $D M$ is given by:

$$
D M=\frac{2 A_{a m}}{A_{a}+A_{m}}
$$




\begin{tabular}{|c|c|c|}
\hline method & mean (DM) & std (DM) \\
\hline with field prior & 0.88 & 0.08 \\
\hline without field prior & 0.657 & 0.0142 \\
\hline
\end{tabular}

Table 1. Statistics of the $D M$ over 20 subject datasets with and without the proposed field prior.

Table 1 reports $D M$ statistics, over 20 subject datasets with and without the proposed field prior $(\alpha \neq 0$ and $\alpha=0$ respectively). The proposed field prior improved significantly the results; it led to a much higher average $D M$.

\section{CONCLUSION AND FUTURE WORK}

Most of the variational segmentation algorithms of LV are based on heavy computation such as distribution matching or shape prior, which lead to computationally expensive segmentation and tracking algorithms. We proposed a new prior that eliminates the need to estimate the distribution or the dependency on a shape prior. The mean prior is based on the consistency of the mean logarithm of intensity inside the endo and epi-cardium in cardiac MR images. We investigated a semi-automatic variational method, implemented using level-set, to track the LV boundaries in MR cardiac sequences by minimizing a functional based on the prior. The curve evolution equations for both endo and epi-cardium obtained using the Euler-Lagrange minimizing of the proposed functional. This method speeds up curve evolution significantly, and reduces computation load. Quantitative statistics showed the conformity of the results with manual segmentation obtained by an expert over 20 subjects dataset.

\section{REFERENCES}

[1] Lee, H. Y., Codella, N., Cham, M., Weinsaft, J., and Wang, Y., "Automatic left ventricle segmentation using iterative thresholding and an active contour model with adaptation on short-axis cardiac mri," IEEE Transactions on Biomedical Engineering 57(4), 905 -913 (2010).

[2] Pednekar, A., Muthupillai, R., Lenge, V. V., Kakadiaris, I. A., and Flamm, S. D., "Automatic identification of the left ventricle in cardiac cine-mr images: Dual-contrast cluster analysis and scout-geometry approaches," Journal of Magnetic Resonance Imaging 23(5), 641-651 (2006).

[3] Lynch, M., Ghita, O., and Whelan, P., "Left ventricle myocardium segmentation using a coupled level-set with a priori knowledge," Computerized Medical Imaging and Graphics 30(4), 255-262 (2006).

[4] Zhou, Z., You, J., Heng, P. A., and Xia, D., "cardiac mr image segmentation and left ventricle surface reconstruction based on level set method," Medicine Meets Virtual Reality: The Magical Next Becomes the Medical , 629-632 (2005). 
[5] Geuns, R. J. M. V., Baks, T., Gronenschild, E. H. B. M., Aben, J. P. P. A. W., Cademartiri, F., and Feyter, P. J. d., "Automatic quantitative left ventricular analysis of cine mr images by using three-dimensional information for contour detection," Radiology 240(1), 215-221 (2006).

[6] Jolly, M. P., "Automatic segmentation of the left ventricle in cardiac mr and ct images," International Journal in Computer Vision 70(2), 151-163 (2006).

[7] Ben Ayed, I., Ross, I., and Li, S., "Embedding overlap priors in variational left ventricle tracking," IEEE Transaction on Medical Imaging 28(12), 1902-1913 (2009).

[8] Corsi, C., Lamberti, C., Battani, R., Maggioni, A., Discenza, G., Eneaney, P. M., Avi, V. M., Lang, R. M., and Caiani, E. G., "Computerized quantification of left ventricular volumes on cardiac magnetic resonance images by level set method," Computer Assisted Radiology and Surgery 1268, 1114 - 1119 (2004).

[9] Sun, W., Cetin, M., Chan, R., Reddy, V., Holmvang, G., Ch, V., and Willsky, A., "Segmenting and tracking the left ventricle by learning the dynamics in cardiac images," Information Processing in Medical Imaging 3565, 553-565 (2005).

[10] Freedman, D., Radke, R. J., Zhang, T., Jeong, Y., Lovelock, D. M., and Chen, G. T. Y., "Model-based segmentation of medical imagery by matching distributions," IEEE Transaction on Medical Imaging 24, 281-292 (2005).

[11] Ben Ayed, I., Li, S., and Ross, I., "A statistical overlap prior for variational image segmentation," International Journal of Computer Vision 85(1), 115-132 (2009).

[12] Cremers, D., Rousson, M., and Deriche, R., "A review of statistical approaches to level set segmentation: Integrating color, texture, motion and shape," International Journal of Computer Vision 72(2), 195-215 (2007).

[13] Cootes, T., Edwards, G., and Taylor, C., "Active appearance models," Proceedings of the European Conference on Computer Vision 2, 484-498 (1998).

[14] Yang, L., Georgescu, B., Zheng, Y., Foran, D. J., and Comaniciu, D., "A fast and accurate tracking algorithm of left ventricles in 3d echocardiography," IEEE international symposium on biomedical imaging , $221-224$ (2008).

[15] Lynch, M., Ghita, O., and Whelan, P., "Left ventricle myocardium segmentation using a coupled level-set with a priori knowledge," Computerized Medical Imaging and Graphics 30(4), 255 - 262 (2006). 